\title{
Brain Diffusion Changes in Emerging Psychosis and the Impact of State- Dependent Psychopathology
}

\author{
André Schmidt ${ }^{\mathrm{a}, \mathrm{b}} \quad$ Claudia Lenz $^{\mathrm{b}}$ Renata Smieskova ${ }^{\mathrm{b}}$ Fabienne Harrisberger ${ }^{\mathrm{b}}$ \\ Anna Walter ${ }^{\mathrm{b}}$ Anita Riecher-Rössler ${ }^{\mathrm{b}}$ Andor Simon ${ }^{\mathrm{b}}$ Undine E. Lang ${ }^{\mathrm{b}}$ \\ Philip McGuire ${ }^{a}$ Paolo Fusar-Polia Stefan J. Borgwardt ${ }^{a, b, c}$ \\ aDepartment of Psychosis Studies, Institute of Psychiatry, Psychology and Neuroscience King's College \\ London, London, UK; 'bepartment of Psychiatry (UPK), University of Basel, Basel, Switzerland; \\ 'Medical Image Analysis Centre, University Hospital Basel, Basel, Switzerland
}

\section{Key Words}

Psychosis • High-risk • First-episode psychosis • DTI • Fractional anisotropy • Mean diffusivity - TBSS • Psychopathology • Antipsychotics

\begin{abstract}
Background/Aims: Previous diffusion tensor imaging (DTI) studies have shown microstructural changes in the brain white matter of at-risk mental state (ARMS) subjects for psychosis and patients with first-episode psychosis (FEP). However, only a few studies have been conducted in clinical high-risk samples and findings in both groups are inconsistent, in particular along the superior longitudinal fasciculus (SLF). Methods: This DTI study used tract-based spatial statistics (TBSS) to compare fractional anisotropy (FA) and mean diffusivity (MD) between ARMS subjects, untreated and antipsychotic-treated FEP patients and healthy controls $(\mathrm{HC})$ across the whole brain and the SLF. Results: Compared to HC, ARMS and FEP patients showed increased FA and decreased MD in diverse regions across the whole brain including the SLF. FA in the SLF was positively correlated with positive psychotic symptoms in ARMS and FEP individuals. Furthermore, untreated but not treated FEP patients showed increased FA in the left inferior longitudinal fasciculus and right SLF. Conclusion: This study revealed increased FA and decreased MD in early stages of psychosis in widespread white matter tracts including the SLF. Our findings further suggest that microstructural changes in the SLF are probably related to state-dependent psychopathology.
\end{abstract}




\section{SiqínAis}

\begin{tabular}{l} 
Neurosignals 2015;23:71-83 \\
\begin{tabular}{l|l}
\hline DOI: 10.1159/000442605 & C 2015 The Author(s). Published by S. Karger AG, Basel \\
Published onIIne: December 19, 2015 & www.karger.com/nsg
\end{tabular} \\
\hline
\end{tabular}

Schmidt et al.: Brain Diffusion Changes in Emerging Psychosis

\section{Introduction}

In recent years, there has been increasing interest in populations at high clinical risk of developing psychosis [1]. This research strategy offers a promising approach to establishing markers for the early detection of the illness and to tailor preventive interventions. These subjects with an at-risk mental state (ARMS) have a high probability of transitioning to overt psychosis (mostly schizophrenia spectrum disorders [2]) within a short period (36\% within 3 years of presentation) [3]. Brain imaging may provide a powerful tool to improve the specificity and validity of an ARMS diagnosis and to validate potential interventions prior to the onset of illness [4]. Although no reliable neuroimaging marker of any clinical utility in the psychosis spectrum has yet been established, studies have repeatedly indicated that abnormalities in brain connectivity may reflect a core pathophysiological mechanism for the onset of psychosis [5, 6].

Diffusion tensor imaging (DTI) is a technique that can be used to detect microstructural changes in brain's white matter [7]. Previous DTI investigations reported that these changes may reflect a critical pathophysiological marker for schizophrenia [8]. Moreover, studies also revealed that abnormal white matter integrity between different brain regions is already evident before the onset of psychosis and further develops along the psychosis continuum [9], which suggests that the assessment of white matter integrity may help to detect the transition from the high-risk state to established psychosis. The most commonly studied metrics in clinical DTI studies are fractional anisotropy (FA) and mean diffusivity (MD). FA measures the diffusion directionality of water molecules and is highest in compact white matter tracts, whereas MD is a measure of total water diffusion averaged over all directions (for a review see [10]). Previous studies have consistently detected alterations in long association fibres, such as the superior longitudinal fasciculus (SLF), in early stages of psychosis, indicating that this may be the most vulnerable pathway that can influence the onset of the disease [11]. The SLF connects the frontal and parietal regions, among other regions, which are crucial for appropriate cognitive functioning [12]. Cognitive deficits as working memory impairments precede the onset of psychosis and may therefore provide valuable predictions of the course of the disease [13]. Evidence from functional magnetic resonance imaging has shown that modulation of fronto-parietal connectivity during working memory processing was gradually reduced from healthy controls to ARMS subjects and then to first-episode patients (FEP) [14], suggesting that fronto-parietal connectivity changes in psychosis evolve along a dynamic trajectory, emerging before disease onset and developing with ongoing illness. DTI studies have shown reduced FA in the SLF in patients with FEP [15], as well as in subjects at clinical $[16,17]$ or genetic high-risk of psychosis [18, 19] (for a review see [11]). DTI studies have also observed increased MD in the SLF of genetic [18] and clinical high-risk individuals [17].

However, these findings seem inconclusive as several studies also reported increased FA in the SLF across different stages of psychosis [20,21], whereas others found no changes [17, $22,23]$. A very recent DTI study in a large sample of drug-naïve FEP patients found increased FA and decreased MD in the SLF [24]. The inconsistency of these DTI findings may be caused by the heterogeneity of the clinical samples [25], the different approaches to DTI analysis and the effects of treatment such as antipsychotic and antidepressant medication. Furthermore, it is important to note that several reports demonstrated a positive correlation between FA in the SLF and positive psychotic symptoms in schizophrenia patients [26-29], which indicates that FA in this pathway may be associated with state-related psychopathology.

In this study, we explored whether FA and MD were differentially expressed along the psychosis continuum. In particular, we used tract-based spatial statistics (TBSS) [30] to compare FA and MD between ARMS subjects, FEP patients (first treated as one group) and healthy controls across the whole brain and the SLF in particular. Findings were controlled for age, gender, antipsychotic and antidepressant medication, all of which are known to 
Schmidt et al.: Brain Diffusion Changes in Emerging Psychosis

significantly affect DTI metrics [15, 31-33]. In a subsequent analysis, we explicitly addressed the effect of antipsychotic medication in FEP patients by comparing HC with untreated and antipsychotic-treated FEP patients only. In accordance with recent evidence [16, 17, 34], we expected that FA would be decreased and MD increased in ARMS and FEP subjects relative to healthy controls and that FA in antipsychotic-treated FEP patients would be increased compared to untreated patients [15]. We further hypothesised that in ARMS and FEP individuals there would be a positive correlation between FA values in the SLF and positive psychotic symptoms [26-29].

\section{Materials and Methods}

\section{Patients}

We recruited 24 healthy controls (HC), 28 ARMS and 37 FEP subjects in our specialised clinic for the early detection of psychosis at the Department of Psychiatry, University of Basel (UPK), Switzerland. All participants provided written informed consent, and the study had research ethics committee permission.

We assessed subjects using the 'Basel Screening Instrument for Psychosis' (BSIP) [35], the Brief Psychiatric Rating Scale (BPRS) [36], the Scale for the Assessment of Negative Symptoms (SANS) [37] and the Global Assessment of Functioning (GAF). We additionally obtained current and previous psychotropic medication, as well as nicotine and illegal drug consumption, by using a semi-structured interview adapted from the Early Psychosis Prevention and Intervention Centre Drug and Alcohol Assessment Schedule (www. eppic.org.au). These exclusion criteria were applied to all groups: history of previous psychotic disorder; psychotic symptomatology secondary to an organic disorder; substance abuse according to ICD-10 research criteria; psychotic symptomatology associated with an affective psychosis or a borderline personality disorder; age under 18 years; inadequate knowledge of the German language; and IQ less than 70, measured with the "Mehrfachwahl-Wortschatz-Test Form B" (MWT-B) [38]. Besides clinical exclusion criteria, 4 ARMS subjects and 14 FEP patients had to be excluded due to MRI artefacts.

Inclusion required one or more of the following: a) attenuated psychotic-like symptoms, b) brief limited intermittent psychotic symptoms (BLIPS) or c) a first or second degree relative with a psychotic disorder plus at least two further risk factors for or indicators of beginning psychosis according to the BSIP screening instrument. Inclusion because of attenuated psychotic symptoms required that change in mental state had to be present at least several times a week and for more than 1 week (a score of 2 or 3 on the BPRS hallucination item, or 3 or 4 on BPRS items for unusual thought content or suspiciousness). Inclusion because of BLIPS required scores of 4 or above on the hallucination item, or 5 or above on the unusual thought content, suspiciousness or conceptual disorganisation items of the BPRS, with each symptom lasting less than one week before resolving spontaneously. After the baseline assessment, the ARMS subjects were followed up clinically and received standard psychiatric case management. At the time of writing, 5 of 24 included ARMS individuals have transited to psychosis (transition rate 21\%; average duration of clinical follow-up was 33.3 months). All ARMS individuals were antipsychotic-naïve, while 11 were treated with antidepressants ( 1 with fluoxetine, St. John's wort and duloxetine, 2 with venlafaxine and mirtazapine and 3 with escitalopram).

FEP patients were defined as subjects who met the operational criteria for first-episode psychosis according to the ICD-10 or DSM-IV [39], but not yet for schizophrenia [40]. Inclusion required scores of 4 or above on the hallucination item or 5 or above on the unusual thought content, suspiciousness or conceptual disorganisation items of the BPRS [40]. The symptoms had to have occurred at least several times a week and persisted for more than one week. The mean duration between the first screening and DTI scanning in FEP patients was 24 months (SD 33). Thirteen of the included 23 FEP patients were not receiving antipsychotic medication (10 were antipsychotic-naïve, 3 antipsychotic-free). Ten FEP patients were receiving low dose antipsychotic treatment at the time of scanning and of unknown duration (missing data) (6 quetiapine: $25 \mathrm{mg}(\mathrm{n}=2), 100 \mathrm{mg}(\mathrm{n}=2), 300 \mathrm{mg}(\mathrm{n}=1)$ and $1000 \mathrm{mg}(\mathrm{n}=1) ; 2$ olanzapine with $5 \mathrm{mg}(\mathrm{n}=2)$; 1 aripiprazole with $5 \mathrm{mg}$ and 1 clozapine with $300 \mathrm{mg}) .7$ FEP patients were also treated with antidepressants ( 1 with citalopram, sertraline and venlafaxine and 2 with escitalopram and paroxetine). 
We recruited HC from the same geographical area as the other groups. HC had no current psychiatric disorder, no history of psychiatric illness, head trauma, neurological illness, serious medical or surgical illness, substance abuse, and no family history of any psychiatric disorder as assessed by an experienced psychiatrist in a detailed semi-structured clinical interview.

Image acquisition and preprocessing of DTI data

Subjects were examined using a 3T magnetic resonance imaging scanner (Magnetom Verio, Siemens Healthcare, Erlangen, Germany) at Basel University Hospital. DTI data were acquired during 10 minutes, based on a single-shot echo planar imaging sequence with the following parameters: echo time/repetition time $=95 / 9200 \mathrm{~ms}$, field of view $=320 \mathrm{~mm}$ and 54 axial slices of $2.5 \mathrm{~mm}$ slice thickness covering the whole brain with an in-plane resolution of $2.5 \times 2.5 \mathrm{~mm}^{2}$. In addition, a 12-channel radio frequency head coil was used, as well as GRAPPA parallel imaging with an acceleration factor of 2, with phase partial Fourier of 6/8. In total, 30 isotropically distributed diffusion weighted directions with $b$-values of $b=900 \mathrm{~s} / \mathrm{mm}^{2}$ and one single reference image with $\mathrm{b}=0 \mathrm{~s} / \mathrm{mm}^{2}$ were acquired twice as two averages.

DTI analysis was performed using FMRIB Software Library (FSL, version 5.0.7, www.fmrib.ox.ac.uk/ fsl). In a first step, FSLView was used to visually check for potential artefacts in all directions. Directions displaying poor image quality (e.g. pulsation artefacts, venetian blind artefacts, inter-slice and intra-slice intensity artefacts, motion artefacts or extreme signal loss due to distortion issues) were subsequently excluded from further analysis. When subjects presented more than $10 \%$ bad directions, the complete data set was excluded from further image processing. For this reason, 4 ARMS and 14 FEP patients had to be consistently excluded from our analysis. Preprocessing was done with the FMRIB's Diffusion Toolbox. DTI data were corrected for effects of motion and image distortions due to eddy currents, by using an affine registration to the $\mathrm{b}=0 \mathrm{~s} / \mathrm{mm}^{2}$ volume [41]. $\mathrm{b}$-vectors were rotated according to the eddy current correction matrix. A brain mask was then created to separate brain from non-brain tissue with the Brain Extraction Tool, and DTIFIT was used to reconstruct the diffusion tensor model at each pixel and extract the FA and MD values.

Tract-based spatial statistics (TBSS)

TBSS was applied for voxel-wise analysis of DTI data [30]. In brief, FA images were first aligned to the Montreal Neurological Institute 152 space, using nonlinear transformation and affine registration. In a further step, the mean FA image and skeleton were created, while the skeleton represents the alignment invariant diffusion tracts common to the subjects included. In the last step, the mean FA skeleton was thresholded at 0.2 and each subject's aligned FA data was projected onto this skeleton. These TBSS steps were analogously repeated for MD values.

\section{Between-group statistical analysis}

For voxel-wise comparisons of FA and MD values across the 3 groups (HC, ARMS and all FEP patients together), we used a 1-way analysis of variance with cluster-based permutation (5000) tests (Randomise [42]). A family-wise error (FWE) correction for multiple comparisons was performed, and threshold-free cluster enhancement was implemented [43]. Given that Randomise corresponds to a 1-sided t test, we considered findings at $\mathrm{p}<0.025$ as significant in order to test 2 -sided. We included age, gender, antipsychotic and antidepressant medication as covariates in the statistical analysis, all of which are known to significantly affect DTI metrics [15, 31-33]. Group comparisons were performed across the whole brain and the SLF bilaterally. For the latter approach, we created a binary mask of the FA/MD skeleton with the SLF tract taken from the JHU White Matter Tractography Atlas [44]. To explicitly probe differences between HC, untreated $(\mathrm{n}=13)$ and treated $(\mathrm{n}=10)$ FEP patients, a subsequent TBSS and randomise analysis was performed. Finally, partial correlation analysis with the control for age, gender and antidepressant medication was used to test the relations between FA values in the SLF and positive psychotic symptoms (indexed with the BPRS items 9, 10, 11 and 15) in ARMS subjects. In FEP patients, correlations were controlled for age, gender, antidepressant and antipsychotic medication. Cook's distance test was used to control for outliners for each correlation analysis (one FEP outliner). 


\section{Results}

\section{Demographic and Clinical Characteristics}

Subjects were well matched for age, handedness, years of education, cigarette smoking and alcohol and cannabis consumption. They differed significantly in IQ, gender, BPRS and SANS total score and in the GAF score. Compared to controls, FEP and ARMS subjects had higher BPRS and SANS scores and reduced GAF scores. FEP patients further had higher BPRS and lower GAF scores compared to ARMS subjects (see Table 1).

\section{Whole Brain TBSS results}

FA differed significantly between HC, ARMS and FEP patients (treated as one group) across the whole brain. Post-hoc testing showed that ARMS individuals exhibited increased FA in several brain regions, with the most affected tracts including the right anterior thalamic radiation, left inferior fronto-occipital fasciculus, superior longitudinal fasciculus and uncinate fasciculus compared with HC (Fig. 1A). FEP patients revealed increased FA in the left inferior longitudinal fasciculus relative to HC (Fig. 1B). No tracts with decreased FA in ARMS and FEP subjects were found compared to HCs.

Comparison of MD across these three groups showed significant differences in all tracts of the brain. In particular, $t$ tests demonstrated significantly decreased MD in ARMS subjects in various brain regions, with the most affected tracts including the left inferior longitudinal fasciculus, inferior fronto-occipital fasciculus, superior longitudinal fasciculus and the right Inferior longitudinal fasciculus compared with HC (Fig. 1C). No tracts with increased MD in ARMS were found compared with HC and no differences between FEP and HC at all. For details of regions see Table 2 .

Table 1. Clinical and demographic characteristics of the study sample

\begin{tabular}{|c|c|c|c|c|}
\hline & $\begin{array}{l}\text { Healthy controls } \\
(\mathrm{n}=24) \\
\text { Mean } \pm S D\end{array}$ & $\begin{array}{l}\text { At-risk mental state } \\
(\mathrm{n}=24) \\
\text { Mean } \pm S D\end{array}$ & $\begin{array}{l}\text { First-episode psychosis } \\
(\mathrm{n}=23) \\
\text { Mean } \pm S D\end{array}$ & Statistics \\
\hline Age (years) & $27.75 \pm 4.59$ & $25.42 \pm 6.74$ & $28.99 \pm 6.47$ & $F=2.152 ; p=0.124$ \\
\hline Gender (female/male) & $14 / 10$ & $6 / 18$ & $7 / 16$ & $\chi^{2}=6.49 ; p=0.039$ \\
\hline Handedness (right) & 22 & 22 & 21 & $\chi^{2}=2.39 ; p=0.665$ \\
\hline Education (years) & $15.38 \pm 2.92$ & $15.04 \pm 3.39$ & $14.48 \pm 3.29$ & $F=0.469 ; p=0.628$ \\
\hline MWT-B (premorbid IQ) & $120 \pm 11.06$ & $115 \pm 14.27$ & $109 \pm 15.80$ & $\begin{array}{l}F=3.837 ; p=0.026 \\
H C>F E P(p=0.008)\end{array}$ \\
\hline BPRS total score & $30.92 \pm 14.42$ & $37.88 \pm 7.64$ & $46.96 \pm 12.52$ & $\begin{array}{l}\mathrm{F}=10.78 ; \mathrm{p}<0.001 \\
\text { FEP }>\text { HC }(\mathrm{p}<0.001) \\
\text { ARMS }>\text { HC }(\mathrm{p}=0.046) \\
\text { FEP }>\text { ARMS }(\mathrm{p}=0.011)\end{array}$ \\
\hline SANS total score & 0 & $24.33 \pm 14.20$ & $18.43 \pm 15.45$ & $\begin{array}{l}F=26.52 ; p<0.001 \\
F E P>H C(p<0.001) \\
\text { ARMS }>\text { HC }(p<0.001)\end{array}$ \\
\hline GAF total score & $88.63 \pm 4.39$ & $68.75 \pm 11.8$ & $60.13 \pm 17.37$ & $\begin{array}{l}\mathrm{F}=33.39 ; \mathrm{p}<0.001 \\
\mathrm{HC}>\mathrm{FEP}(\mathrm{p}<0.001) \\
\mathrm{HC}>\text { ARMS }(\mathrm{p}<0.001) \\
\text { ARMS }>\text { FEP }(\mathrm{p}=0.019)\end{array}$ \\
\hline Cigarettes smoked per day & $4.08 \pm 7.01$ & $6.00 \pm 8.20$ & $9.54 \pm 10.75$ & $F=2.34 ; p=0.104$ \\
\hline Number of subjects on antipsychotics & 0 & 0 & 13 & $\chi^{2}=24.291 ; p<0.001$ \\
\hline Number of subjects on antidepressants & 0 & 11 & 7 & $\chi^{2}=27.265 ; p<0.001$ \\
\hline $\begin{array}{l}\text { Alcohol consumption } \\
\text { None/moderate/uncontrolled }\end{array}$ & $1 / 21 / 2$ & $4 / 18 / 2$ & $4 / 16 / 3$ & $\chi^{2}=2.942 ; p=0.568$ \\
\hline Number of subjects consuming cannabis & 4 & 5 & 7 & $\chi^{2}=1.335 ; p=0.513$ \\
\hline
\end{tabular}

HC, healthy controls; FEP, first-episode psychosis; ARMS, at-risk mental state; MWT-B, Mehrfachwahl-Wortschatz-Test Form B; (Multiple Choice Vocabulary Test); BPRS, Brief Psychiatric Rating Scale; SANS, Scale for the Assessment of Negative Symptoms; GAF, Global Assessment of Functioning 


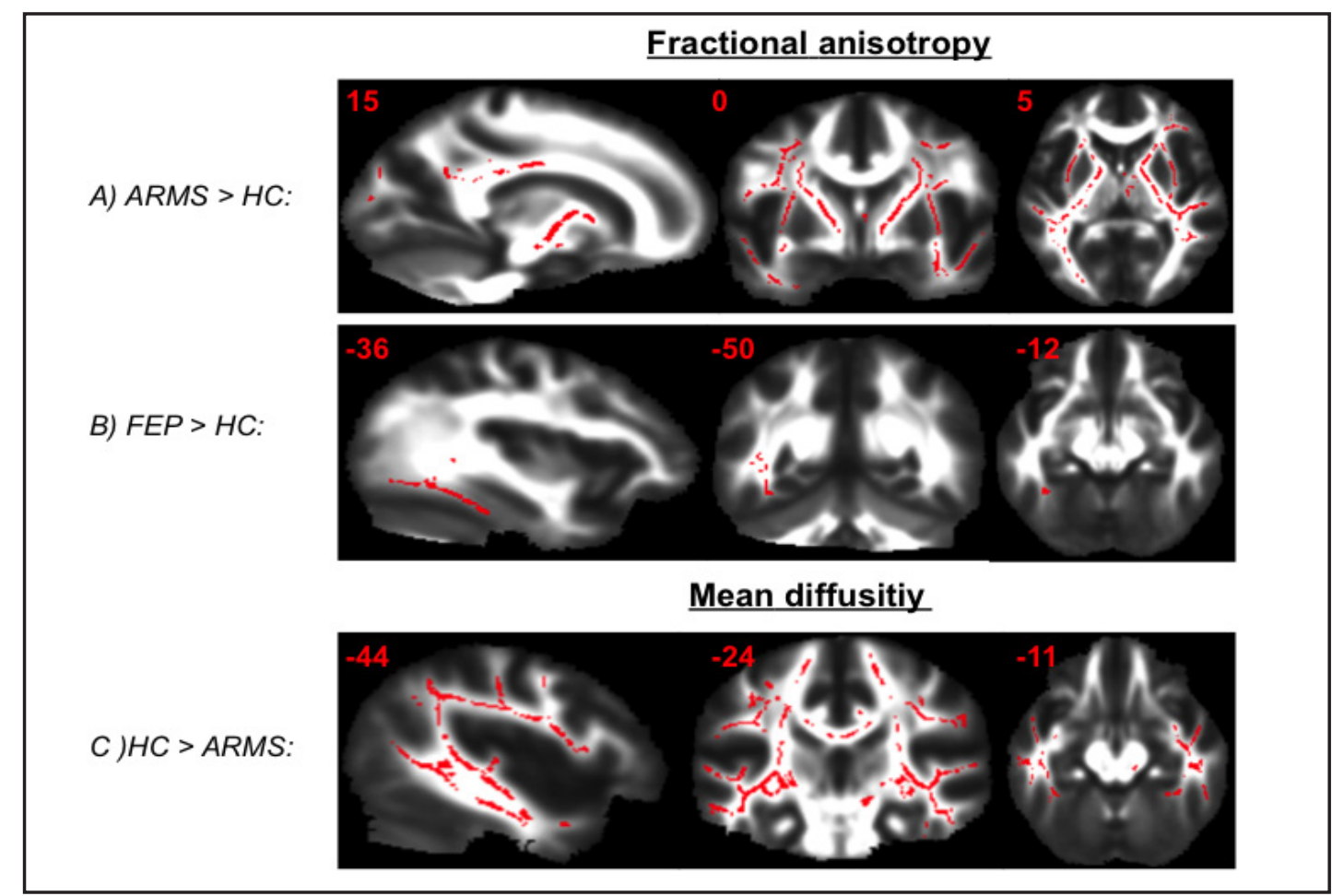

Fig. 1. Group differences between HC, ARMS and FEP patients in fractional anisotropy (A, B) and mean diffusivity (C) across the whole brain. Significance threshold was set to $p<0.025$, FWE-corrected. Group differences are displayed on a mean FA image obtained from TBSS over all groups. Red numbers at the top reflect $\mathrm{x}, \mathrm{y}$ and $\mathrm{z}$ coordinates in the Montreal Neurological Institute space. Left side on figure corresponds to left hemisphere.

Table 2. Significant TBSS whole brain results for FA and MD values across all groups

\begin{tabular}{|c|c|c|c|c|c|c|c|c|}
\hline $\begin{array}{l}\text { DTI } \\
\text { Parameter } \\
\end{array}$ & Comparison & $\begin{array}{l}\text { Cluster } \\
\text { Number }\end{array}$ & $\begin{array}{l}\text { Voxel } \\
\text { number }\end{array}$ & MAX 1-p & MAXX & MAXY & MAXZ & Structures to which each cluster peak belongs to* \\
\hline \multirow[t]{7}{*}{ FA } & \multirow[t]{5}{*}{ ARMS $>\mathrm{HC}$} & 5 & 12344 & 0.998 & 15 & 0 & 5 & Anterior thalamic radiation $\mathrm{R}$ \\
\hline & & 4 & 10731 & 0.992 & -33 & 6 & 0 & $\begin{array}{l}\text { Inferior fronto-occipital fasciculus L Superior longitudinal } \\
\text { fasciculus L, Uncinate fasciculus L }\end{array}$ \\
\hline & & 3 & 249 & 0.976 & 22 & -79 & 18 & $\begin{array}{l}\text { Forceps major, Inferior fronto-occipital fasciculus R, } \\
\text { Inferior longitudinal fasciculus R }\end{array}$ \\
\hline & & 2 & 70 & 0.975 & 3 & -25 & 14 & Anterior thalamic radiation $\mathrm{R}$ \\
\hline & & 1 & 62 & 0.975 & 23 & -84 & 8 & Forceps major, Inferior fronto-occipital fasciculus $R$ \\
\hline & $\mathrm{FEP}>\mathrm{HC}$ & 1 & 638 & 0.98 & -36 & -50 & -12 & Inferior longitudinal fasciculus L \\
\hline & $\mathrm{HC}>\mathrm{ARMS}$ & 2 & 20902 & 0.997 & -44 & -24 & -11 & $\begin{array}{l}\text { Inferior longitudinal fasciculus L, Inferior fronto-occipital fasciculus L, } \\
\text { Superior longitudinal fasciculus L, Superior longitudinal fasciculus } \\
\text { (temporal part) L }\end{array}$ \\
\hline MD & & 1 & 19705 & 0.998 & 43 & -9 & -20 & Inferior longitudinal fasciculus $\mathrm{R}$ \\
\hline
\end{tabular}

* only tract with $>5 \%$ probability are included in the labels, tracts with the highest probabilities were listed first. We only report tracts including $\geq 10$ voxels. Labels are provided in accordance with JHU White Matter Tractography Atlas [44].

\section{SLF tracts}

FA in the right and left SLF differed significantly between HC, ARMS and all FEP patients. Subsequent post-hoc testing showed that ARMS individuals had increased FA in the right and left hemisphere compared with HC (Fig. 2A and B). FEP patients revealed no differences in the SLF compared with HCs. There were also significant inter-group MD differences in the right and left SLF. Post-hoc analysis showed decreased MDs in ARMS and FEP subjects in the right SLF compared with HCs (Fig. 2C and D). ARMS subjects had also decreased MD in the left SLF relative to HC (Fig. 2E). No increase in MD was found in ARMS and FEP subjects 


\section{SIGENAis}

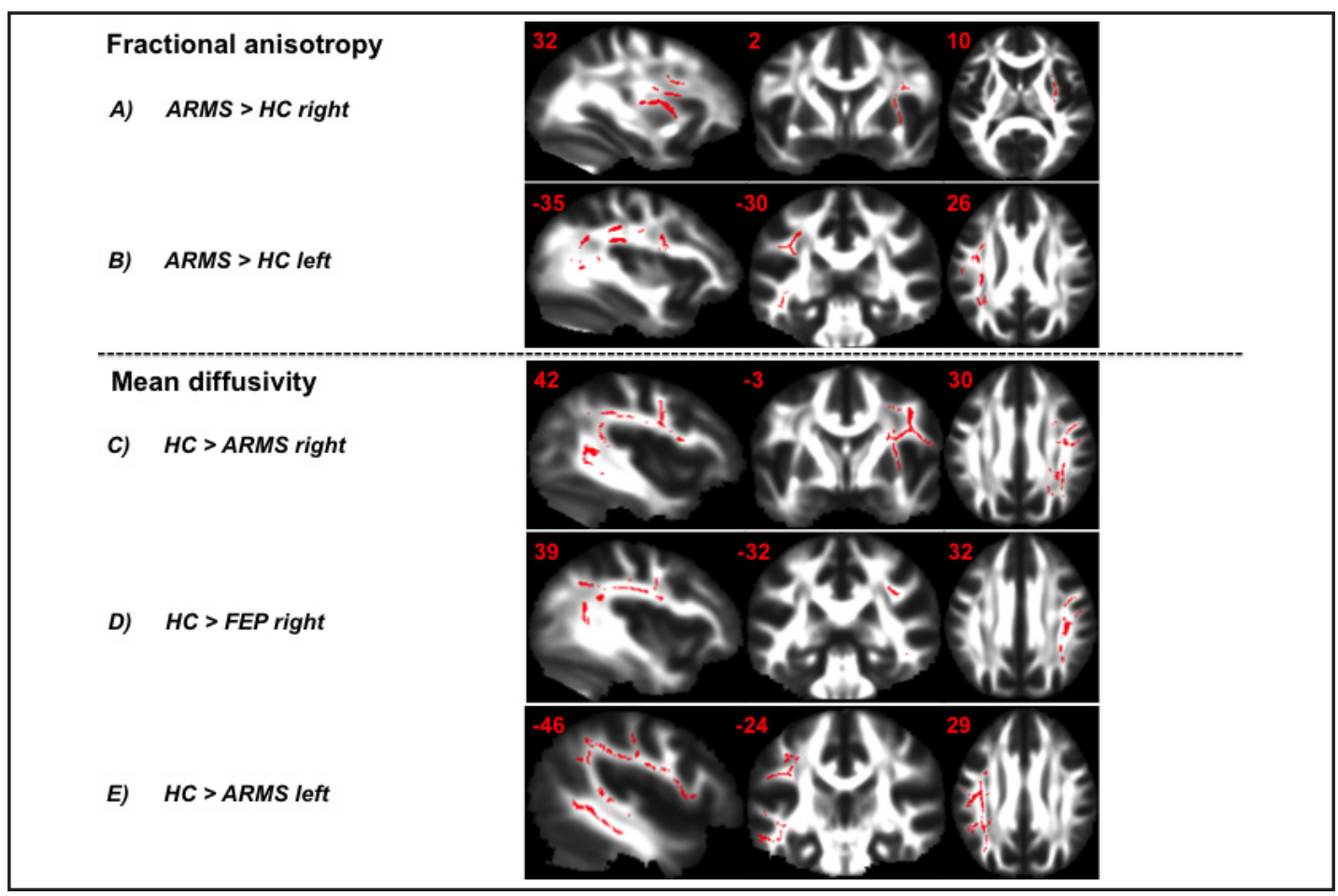

Fig. 2. Group differences between HC, ARMS and FEP patients in fractional anisotropy (A, B) and mean diffusivity (C, D, E) along the SLF. Significance threshold was set to $p<0.025$, FWE-corrected. Group differences are displayed on a mean FA image obtained from TBSS over all groups. Red numbers at the top reflect $\mathrm{x}, \mathrm{y}$ and $\mathrm{z}$ coordinates in the Montreal Neurological Institute space. Left side on figure corresponds to left hemisphere.

Table 3. Significant clusters along the SLF obtained from TBSS for FA and MD across all groups

\begin{tabular}{|c|c|c|c|c|c|c|c|c|}
\hline DTI Parameter & $\begin{array}{l}\text { SLF } \\
\text { Hemisphere }\end{array}$ & Comparison & $\begin{array}{l}\text { Cluster } \\
\text { Number }\end{array}$ & $\begin{array}{l}\text { Voxel } \\
\text { number }\end{array}$ & MAX 1-p & MAXX & MAX Y & $\operatorname{MAX} Z$ \\
\hline \multirow[t]{9}{*}{$\mathrm{FA}$} & \multirow[t]{4}{*}{ Right } & \multirow[t]{4}{*}{ ARMS > HC } & 4 & 305 & 0.989 & 32 & 2 & 10 \\
\hline & & & 3 & 231 & 0.984 & 32 & 12 & 25 \\
\hline & & & 2 & 30 & 0.98 & 27 & -11 & 22 \\
\hline & & & 1 & 14 & 0.976 & 32 & -13 & 11 \\
\hline & \multirow[t]{5}{*}{ Left } & \multirow[t]{5}{*}{ ARMS > HC } & 5 & 881 & 0.995 & -35 & -30 & 26 \\
\hline & & & 4 & 705 & 0.993 & -39 & -50 & 13 \\
\hline & & & 3 & 277 & 0.985 & -43 & -25 & -10 \\
\hline & & & 2 & 124 & 0.992 & -43 & -15 & -20 \\
\hline & & & 1 & 20 & 0.978 & -36 & -61 & 13 \\
\hline \multirow[t]{5}{*}{ MD } & \multirow[t]{4}{*}{ Right } & \multirow[t]{2}{*}{$\mathrm{HC}>\mathrm{ARMS}$} & 2 & 4346 & 0.999 & 42 & -3 & 30 \\
\hline & & & 1 & 369 & 0.989 & 33 & -4 & 10 \\
\hline & & \multirow[t]{2}{*}{$\mathrm{HC}>\mathrm{FEP}$} & 2 & 1468 & 0.99 & 39 & -32 & 32 \\
\hline & & & 1 & 183 & 0.982 & 48 & -37 & -8 \\
\hline & Left & HC $>$ ARMS & 1 & 5031 & 0.999 & -46 & -24 & 29 \\
\hline
\end{tabular}

We only report tracts including $\geq 10$ voxels.

relative to HCs. For details of clusters with respect to differences in FA and MD along the SLF, see Table 3.

\section{Effect of antipsychotic medication}

Comparing HC, untreated and antipsychotic-treated FEP patients across the whole brain, we found that untreated but not antipsychotic-treated FEP patients had increased FA in the 


\section{SIGENAis}

\section{A) Whole brain fractional anisotropy}

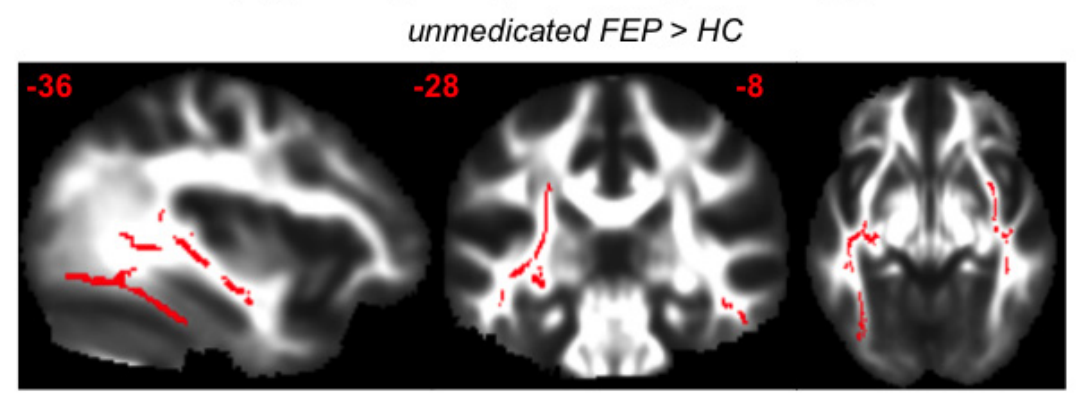

B) Fractional anisotropy along the right SLF

unmedicated FEP > HC

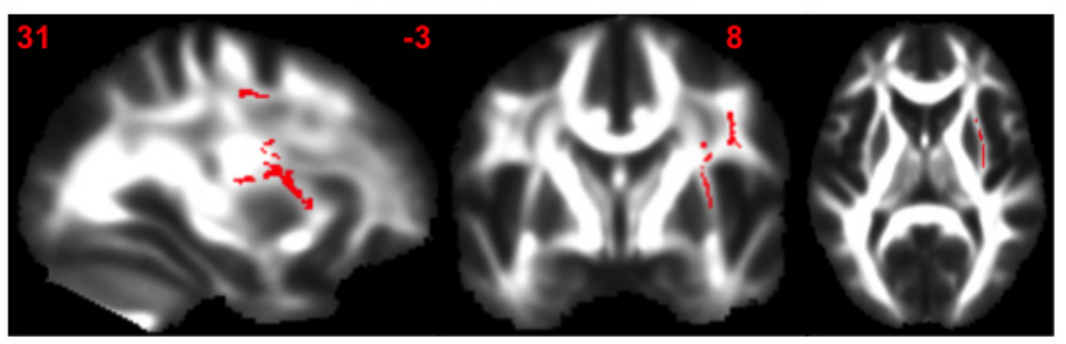

Fig. 3. Group differences between HC, antipsychotic-treated and untreated FEP patients across the whole brain (A) and the SLF (B). Significance threshold was set to $p<0.025$, FWE-corrected.

Table 4. Whole brain comparison between HC, medicated and unmedicated FEP patients

\begin{tabular}{|c|c|c|c|c|c|c|c|c|}
\hline $\begin{array}{l}\text { DTI } \\
\text { Parameter }\end{array}$ & Comparison & $\begin{array}{l}\text { Cluster } \\
\text { Number }\end{array}$ & $\begin{array}{l}\text { Voxel } \\
\text { Number }\end{array}$ & MAX 1-p & $\operatorname{MAXX}$ & MAXY & MAX Z & $\begin{array}{l}\text { Structures to which each cluster } \\
\text { peak belongs to* }\end{array}$ \\
\hline \multirow[t]{5}{*}{$\mathrm{FA}$} & \multirow[t]{5}{*}{ FEP-unmedicated $>\mathrm{HC}$} & 5 & 2644 & 0.985 & -39 & -35 & -15 & Inferior longitudinal fasciculus $\mathrm{L}$ \\
\hline & & 4 & 2577 & 0.984 & 33 & 6 & 2 & $\begin{array}{l}\text { Superior longitudinal fasciculus } R \text {, Inferior } \\
\text { fronto-occipital fasciculus R }\end{array}$ \\
\hline & & 3 & 532 & 0.979 & 34 & -6 & 4 & $\begin{array}{l}\text { Superior longitudinal fasciculus R, Superior } \\
\text { longitudinal fasciculus (temporal part) R }\end{array}$ \\
\hline & & 2 & 477 & 0.982 & -31 & 6 & 3 & Uncinate fasciculus $\mathrm{L}$ \\
\hline & & 1 & 379 & 0.978 & -30 & -64 & 18 & $\begin{array}{l}\text { Inferior longitudinal fasciculus L, Inferior } \\
\text { fronto-occipital fasciculus L, Forceps major }\end{array}$ \\
\hline
\end{tabular}

left inferior longitudinal fasciculus and fronto-occipital fasciculus and in the right superior longitudinal fasciculus and inferior fronto-occipital fasciculus relative to HC (Fig. 3A, Table 4). Along the SLF, we found that untreated but not treated FEP patients had increased FA in the right SLF compared with HC (Fig. 3B).

Relation between FA in the SLF and positive psychotic symptoms

In ARMS subjects, FA was positively correlated with positive psychotic symptoms in the right ( $\mathrm{r}=0.530, \mathrm{p}=0.013$, corrected for multiple testing) but not left SLF $(\mathrm{r}=0.308, \mathrm{p}$ $=0.174$ ) (Fig. 4A). In FEP patients, we found a significant positive correlation between FA in the right $(\mathrm{r}=0.690, \mathrm{p}=0.002$, corrected for multiple testing) and left SLF ( $\mathrm{r}=0.670, \mathrm{p}=$ 0.002 , corrected for multiple testing) (Fig. 4B). 


\section{SiGínAis}

Fig. 4. Significant positive correlation between FA in the SLF and positive psychotic symptoms in ARMS (A) and FEP individuals (B).
Neurosignals 2015;23:71-83

\begin{tabular}{|l|l|l|l|l}
\hline DOI: 10.1159/000442605 & (C) 2015 The Author(s). Published by S. Karger AG, Basel
\end{tabular}

Schmidt et al.: Brain Diffusion Changes in Emerging Psychosis

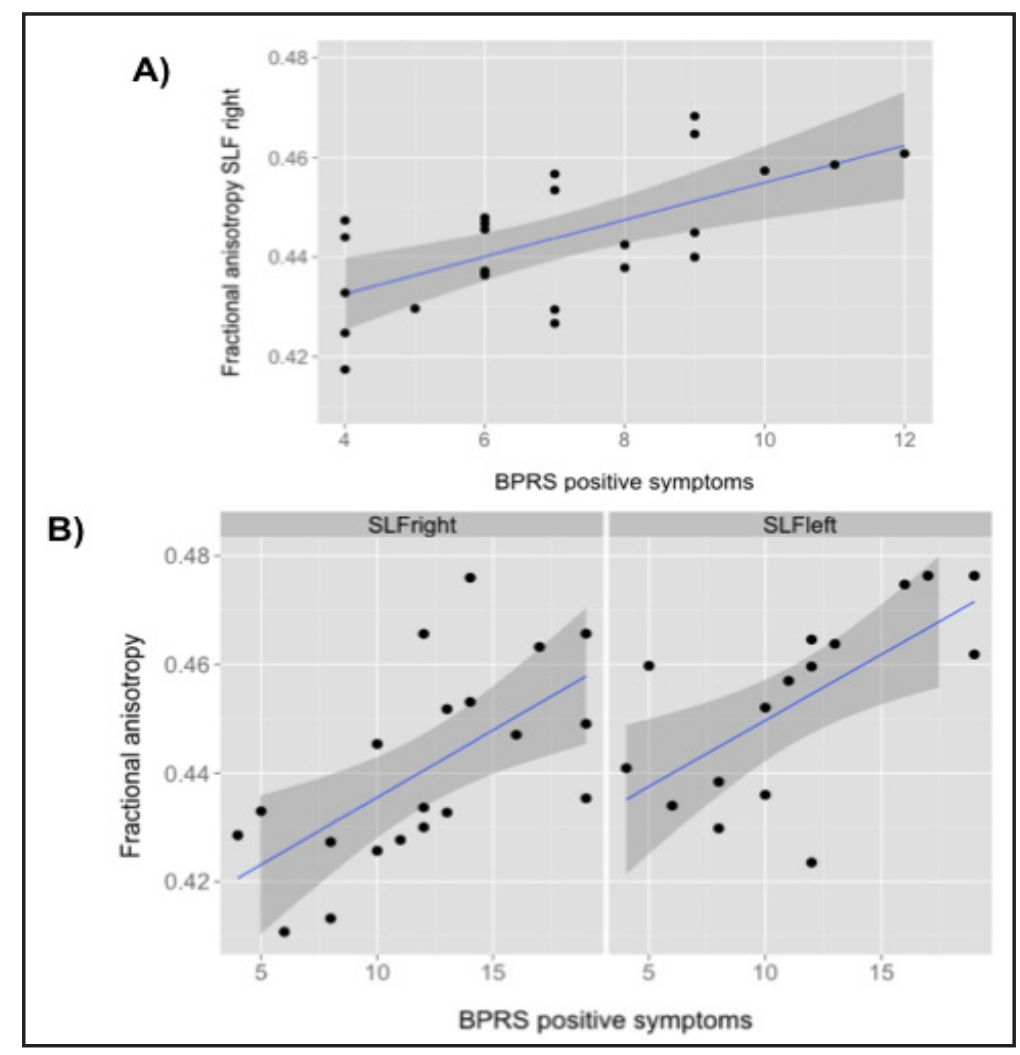

\section{Discussion}

This study explored FA and MD among HC, ARMS and FEP subjects by controlling for the confounding effects of both antipsychotic and antidepressant medication. This is important as both medications can affect FA [15, 33]. Furthermore, to explicitly address antipsychotic medication, a subsequent analysis was also performed to compare HC with antipsychotictreated and untreated FEP patients. Given the high relevance of SLF in emerging psychosis [11], the whole brain analysis was completed by a region of interest analysis along the SLF.

At the whole brain level, we found increased FA in the right anterior thalamic radiation, forceps major, inferior fronto-occipital and longitudinal fasciculus and left inferior frontooccipital fasciculus, uncinate fasciculus and SLF in ARMS subjects relative to HC. Considering the SLF in particular, ARMS individuals exhibited increased FA in the bilateral SLF. These findings are in line with a previous report in genetic high-risk subjects showing increased FA values in the right frontal gyri [20], but contrasts with evidence in clinical high-risk subjects detecting decreased FA in the left SLF [16,31], and the left inferior fronto-occipital fasciculus [16]. There is also a previous study that found no whole brain FA differences in clinical highrisk subjects compared with HC at all [17]. We further found decreased MD in ARMS subjects in the left inferior longitudinal and fronto-occipital fasciculus. There is only one other study that investigated MD in clinical high-risk subjects, which found increased MD in the right SLF [17]. Inconsistencies between studies with high-risk samples may result from different methods of data analysis [45], or depend on whether there was adjustment for different covariates. With respect to the latter point, our DTI findings in ARMS were controlled for age, gender and antidepressants, whereas other studies that reported contrasting FA [16] and MD findings [17] did not adjust for any of these variables [16] or medication effects $[17,31]$. Another important point that complicates comparison between studies is the high clinical heterogeneity of high-risk samples [25]. Furthermore, a crucial issue is the ratio of converters to non-converters in each study, as this may reflect a different level of risk of the 
sample investigated. In our sample, only five of the 24 participants developed a psychosis (by 14.9 months in average), so that we dispensed with a subgroup analysis because of limited power. Thus data from large-scale longitudinal multicentre studies with a higher ratio between converters and non-converters are required to eventually ascertain whether FA is a valuable marker to detect subsequent transition to psychosis.

When controlled for the effect of antipsychotic medication in FEP patients, we found increased FA in the left inferior longitudinal fasciculus compared with HC. We also found decreased MD in the right SLF if the analysis was restricted to this region. This study further indicates that structural integrity along the SLF is probably associated with statedependent psychopathology in emerging psychosis. In particular, we found that FA in the bilateral SLF and right SLF was associated with positive psychotic symptoms in FEP and ARMS individuals, respectively. Consistent with these findings in early stages of psychosis, a previous DTI reported a positive correlation between FA values of the left frontal part of the SLF and the severity score of auditory hallucinations in the hallucinating patient group [26]. Consistently, it has been shown that the propensity to experience auditory hallucinations was associated with relatively increased FA in the SLF in schizophrenia patients $[26,27]$ and that FA in the arcuate fasciculus, a part of the SLF were positively related to positive symptoms in schizophrenia patients [29]. A positive association between positive psychotic symptoms and FA values in the superior and inferior longitudinal fasciculi and inferior frontooccipital fasciculus has also been detected in never-medicated patients with schizophrenia $[28,46]$. Thus our findings extend previous evidence in chronic psychosis by showing that the relation between positive psychotic symptoms and FA in the SLF is already evident in emerging psychosis.

After dissociating antipsychotic-treated from untreated FEP patients, we found increased FA in the left inferior longitudinal fasciculus and right SLF relative to HC only in untreated but not treated patients. The same was evident in the right SLF. It is important to note that we found no differences between treated and untreated FEP patients in accordance with another cross-sectional study [47]. In contrast to our FA findings, no MD differences whether across the whole brain level or the SLF between treated and untreated FEP patients compared to HC were found, may suggesting that FA and MD are modulated differently by antipsychotic treatment. The FA increases in the right SLF of untreated FEP patients resonance with a very recent study in a large sample of in drug-naive FEP patients compared to healthy subjects [24] and with a previous meta-analysis showing that white matter in tracts passing the right SLF is increased in FEP [21]. However, another study in drug-naïve FEP patients has reported decreased FA in the right SLF [48]. This study thus contributes further to the current debate as to whether FA is decreased or increased in psychosis [11, 49], may indicating diverse pathological dynamics of structural connectivity.

Some limitations of this study should be considered. The small samples sizes regarding antipsychotic-treated and untreated FEP together with the cross-sectional design limits interpretation about treatment effects. A recent DTI study showed that FA findings in FEP depended on the size of the studied sample [34]. More longitudinal study designs with large sample and uniform medication strategies, given that atypical and typical antipsychotics may differentially affect myelination in patients [50], are needed to draw robust conclusions on treatment responses. Future studies should also address comorbidities as they could be major sources of hetereogeneity in the biology of high-risk samples [51]. Another limitation is that our groups showed differences in IQ and GAF scores, which may have confounded our DTI findings. Furthermore, we did not explicitly examine group differences along other tracts such as the corticospinal tract or inferior longitudinal fasciculus to control the specificity of our SLF findings. However, the here presented whole brain approach in combination with an a priori defined region of interest analysis of the SLF (with subsequent correlations on those values), both repeated twice to further explore medication effects reflect already an extensive investigation. Finally, we did not acquire T2-weighted images to control for white matter hyperintensities and/or lesions, which might have affected our findings. 


\section{SIGiNAIS}

Neurosignals 2015;23:71-83

\begin{tabular}{l|l}
\hline DOI: 10.1159/000442605 & (c) 2015 The Author(s). Published by S. Karger AG, Basel \\
Published onlIne: December 19, 2015 & www.karger.com/nsg
\end{tabular}

Schmidt et al.: Brain Diffusion Changes in Emerging Psychosis

\section{Conclusion}

This study demonstrated increased FA and decreased MD in early stages of psychosis among widespread white matter tracts including the SLF, which probably depended on stage-related psychopathology. Our findings further support the notion that structural abnormalities in the SLF may be critical for the onset of psychosis [17]. With larger samples sizes together with state-dependent psychopathology, assessment of DTI metrics, either with conventional or more sophisticated network analysis, can be promising, not only for the understanding of the underlying pathophysiology, but may also be used to detect subsequent transition to psychosis or to evaluate treatment interventions.

\section{Acknowledgments}

The authors would like to thank Dr. Markus Klarhöfer, Siemens Switzerland and Radiological Physics, University of Basel Hospital, for providing his help with deducing the correct angulation of b-vectors from the DTI data. The authors acknowledge the contribution of the subjects who took part in this study and they thank the FEPSY study group for recruitment and management of participants. A.S. (No. 155184), R.S. and S.J.B. (No. 3232BO_119382) were supported by the Swiss National Science Foundation.

\section{Disclosure Statement}

The authors declare no conflict of interest.

\section{References}

1 Fusar-Poli P, Borgwardt S, Bechdolf A, Addington J, Riecher-Rössler A, Schultze-Lutter F, Keshavan M, Wood S, Ruhrmann S, Seidman LJ, Valmaggia L, Cannon T, Velthorst E, De Haan L, Cornblatt B, Bonoldi I, Birchwood M, McGlashan T, Carpenter W, McGorry P, Klosterkötter J, McGuire P, Yung A: The psychosis high-risk state: A comprehensive state-of-the-art review. JAMA Psychiatry 2013;70:107-120.

- Fusar-Poli P, Bechdolf A, Taylor MJ, Bonoldi I, Carpenter WT, Yung AR, McGuire P: At risk for schizophrenic or affective psychoses? A meta-analysis of dsm/icd diagnostic outcomes in individuals at high clinical risk. Schizophr Bull 2013;39:923-932.

- 3 Fusar-Poli P, Bonoldi I, Yung AR, Borgwardt S, Kempton MJ, Valmaggia L, Barale F, Caverzasi E, McGuire P: Predicting psychosis: Meta-analysis of transition outcomes in individuals at high clinical risk. Arch Gen Psychiatry 2012;69:220-229.

4 Borgwardt S, Fusar-Poli P: Third-generation neuroimaging in early schizophrenia: Translating research evidence into clinical utility. Br J Psychiatry 2012;200:270-272.

5 Pettersson-Yeo W, Allen P, Benetti S, McGuire P, Mechelli A: Dysconnectivity in schizophrenia: Where are we now? Neurosci Biobehav Rev 2011;35:1110-1124.

6 Dauvermann MR, Whalley HC, Schmidt A, Lee GL, Romaniuk L, Roberts N, Johnstone EC, Lawrie SM, Moorhead TW: Computational neuropsychiatry - schizophrenia as a cognitive brain network disorder. Front Psychiatry 2014;5:30.

7 Basser PJ, Pierpaoli C: Microstructural and physiological features of tissues elucidated by quantitativediffusion-tensor mri. J Magn Reson B 1996;111:209-219.

8 Kubicki M, McCarley R, Westin CF, Park HJ, Maier S, Kikinis R, Jolesz FA, Shenton ME: A review of diffusion tensor imaging studies in schizophrenia. J Psychiatr Res 2007;41:15-30.

-9 Peters BD, Blaas J, de Haan L: Diffusion tensor imaging in the early phase of schizophrenia: What have we learned? J Psychiatr Res 2010;44:993-1004.

$\longrightarrow 10$ Jellison BJ, Field AS, Medow J, Lazar M, Salamat MS, Alexander AL: Diffusion tensor imaging of cerebral white matter: A pictorial review of physics, fiber tract anatomy, and tumor imaging patterns. AJNR Am J Neuroradiol 2004;25:356-369. 
11 Schmidt A, Diwadkar VA, Smieskova R, Harrisberger F, Lang UE, McGuire P, Fusar-Poli P, Borgwardt S: Approaching a network connectivity-driven classification of the psychosis continuum: A selective review and suggestions for future research. Front Hum Neurosci 2014;8:1047.

12 Gazzaley A, Nobre AC: Top-down modulation: Bridging selective attention and working memory. Trends Cogn Sci 2012;16:129-135.

13 Fusar-Poli P, Deste G, Smieskova R, Barlati S, Yung AR, Howes O, Stieglitz RD, Vita A, McGuire P, Borgwardt S: Cognitive functioning in prodromal psychosis: A meta-analysiscognitive functioning in prodromal psychosis. Arch Gen Psychiatry 2012;69:562-571.

-14 Schmidt A, Smieskova R, Aston J, Simon A, Allen P, Fusar-Poli P, McGuire PK, Riecher-Rössler A, Stephan KE, Borgwardt S: Brain connectivity abnormalities predating the onset of psychosis: Correlation with the effect of medication. JAMA Psychiatry 2013;70:903-912.

15 Reis Marques T, Taylor H, Chaddock C, Dell'acqua F, Handley R, Reinders AA, Mondelli V, Bonaccorso S, Diforti M, Simmons A, David AS, Murray RM, Pariante CM, Kapur S, Dazzan P: White matter integrity as a predictor of response to treatment in first episode psychosis. Brain 2014;137:172-182.

16 Carletti F, Woolley JB, Bhattacharyya S, Perez-Iglesias R, Fusar Poli P, Valmaggia L, Broome MR, Bramon E, Johns L, Giampietro V, Williams SC, Barker GJ, McGuire PK: Alterations in white matter evident before the onset of psychosis. Schizophr Bull 2012;38:1170-1179.

17 Clemm von Hohenberg C, Pasternak O, Kubicki M, Ballinger T, Vu MA, Swisher T, Green K, Giwerc M, Dahlben B, Goldstein JM, Woo TU, Petryshen TL, Mesholam-Gately RI, Woodberry KA, Thermenos HW, Mulert C, McCarley RW, Seidman LJ, Shenton ME: White matter microstructure in individuals at clinical high risk of psychosis: A whole-brain diffusion tensor imaging study. Schizophr Bull 2014;40:895-903.

18 Clark KA, Nuechterlein KH, Asarnow RF, Hamilton LS, Phillips OR, Hageman NS, Woods RP, Alger JR, Toga AW, Narr KL: Mean diffusivity and fractional anisotropy as indicators of disease and genetic liability to schizophrenia. J Psychiatr Res 2011;45:980-988.

19 Knöchel C, O'Dwyer L, Alves G, Reinke B, Magerkurth J, Rotarska-Jagiela A, Prvulovic D, Hampel H, Linden DE, Oertel-Knöchel V: Association between white matter fiber integrity and subclinical psychotic symptoms in schizophrenia patients and unaffected relatives. Schizophr Res 2012;140:129-135.

20 Hoptman MJ, Nierenberg J, Bertisch HC, Catalano D, Ardekani BA, Branch CA, Delisi LE: A dti study of white matter microstructure in individuals at high genetic risk for schizophrenia. Schizophr Res 2008;106:115124.

21 Bora E, Fornito A, Radua J, Walterfang M, Seal M, Wood SJ, Yücel M, Velakoulis D, Pantelis C: Neuroanatomical abnormalities in schizophrenia: A multimodal voxelwise meta-analysis and metaregression analysis. Schizophr Res 2011;127:46-57.

22 Steel RM, Bastin ME, McConnell S, Marshall I, Cunningham-Owens DG, Lawrie SM, Johnstone EC, Best JJ: Diffusion tensor imaging (dti) and proton magnetic resonance spectroscopy (1h mrs) in schizophrenic subjects and normal controls. Psychiatry Res 2001;106:161-170.

23 Foong J, Symms MR, Barker GJ, Maier M, Miller DH, Ron MA: Investigating regional white matter in schizophrenia using diffusion tensor imaging. Neuroreport 2002;13:333-336.

24 Filippi M, Canu E, Gasparotti R, Agosta F, Valsecchi P, Lodoli G, Galluzzo A, Comi G, Sacchetti E: Patterns of brain structural changes in first-contact, antipsychotic drug-naive patients with schizophrenia. AJNR Am J Neuroradiol 2014;35:30-37.

25 Simon AE, Umbricht D, Lang UE, Borgwardt S: Declining transition rates to psychosis: The role of diagnostic spectra and symptom overlaps in individuals with attenuated psychosis syndrome. Schizophr Res 2014;159:292-298.

-26 Seok JH, Park HJ, Chun JW, Lee SK, Cho HS, Kwon JS, Kim JJ: White matter abnormalities associated with auditory hallucinations in schizophrenia: A combined study of voxel-based analyses of diffusion tensor imaging and structural magnetic resonance imaging. Psychiatry Res 2007;156:93-104.

27 Shergill SS, Kanaan RA, Chitnis XA, O'Daly O, Jones DK, Frangou S, Williams SC, Howard RJ, Barker GJ, Murray RM, McGuire P: A diffusion tensor imaging study of fasciculi in schizophrenia. Am J Psychiatry 2007;164:467-473.

28 Cheung V, Chiu C, Law C, Cheung C, Hui C, Chan K, Sham P, Deng M, Tai K, Khong P, McAlonan G, Chua $\mathrm{S}$, Chen E: Positive symptoms and white matter microstructure in never-medicated first episode schizophrenia. Psychol Med 2010;41:1709-1719.

-29 Rotarska-Jagiela A, Oertel-Knoechel V, DeMartino F, van de Ven V, Formisano E, Roebroeck A, Rami A, Schoenmeyer R, Haenschel C, Hendler T, Maurer K, Vogeley K, Linden DE: Anatomical brain connectivity and positive symptoms of schizophrenia: A diffusion tensor imaging study. Psychiatry Res 2009;174:9-16. 
Schmidt et al.: Brain Diffusion Changes in Emerging Psychosis

30 Smith SM, Jenkinson M, Johansen-Berg H, Rueckert D, Nichols TE, Mackay CE, Watkins KE, Ciccarelli O, Cader MZ, Matthews PM, Behrens TE: Tract-based spatial statistics: Voxelwise analysis of multi-subject diffusion data. Neuroimage 2006;31:1487-1505.

-31 Karlsgodt KH, Niendam TA, Bearden CE, Cannon TD: White matter integrity and prediction of social and role functioning in subjects at ultra-high risk for psychosis. Biol Psychiatry 2009;66:562-569.

-32 Hsu JL, Leemans A, Bai CH, Lee CH, Tsai YF, Chiu HC, Chen WH: Gender differences and age-related white matter changes of the human brain: A diffusion tensor imaging study. Neuroimage 2008;39:566-577.

33 Korgaonkar MS, Williams LM, Song YJ, Usherwood T, Grieve SM: Diffusion tensor imaging predictors of treatment outcomes in major depressive disorder. Br J Psychiatry 2014;205:321-328.

34 Melicher T, Horacek J, Hlinka J, Spaniel F, Tintera J, Ibrahim I, Mikolas P, Novak T, Mohr P, Hoschl C: White matter changes in first episode psychosis and their relation to the size of sample studied: A dti study. Schizophr Res 2015;162:22-28.

-35 Riecher-Rössler A, Aston J, Ventura J, Merlo M, Borgwardt S, Gschwandtner U, Stieglitz RD: [the basel screening instrument for psychosis (bsip): Development, structure, reliability and validity]. Fortschr Neurol Psychiatr 2008;76:207-216.

-36 Lukoff D, Liberman RP, Nuechterlein KH: Symptom monitoring in the rehabilitation of schizophrenic patients. Schizophr Bull 1986;12:578-602.

37 Andreasen NC: The scale for the assessment of negative symptoms (sans): Conceptual and theoretical foundations. Br J Psychiatry 1989:S49-58.

-38 Lehrl S, Triebig G, Fischer B: Multiple choice vocabulary test mwt as a valid and short test to estimate premorbid intelligence. Acta Neurol Scand 1995;91:335-345.

39 Breitborde NJ, Srihari VH, Woods SW: Review of the operational definition for first-episode psychosis. Early Interv Psychiatry 2009;2009:259-265.

40 Yung AR, Phillips LJ, McGorry PD, McFarlane CA, Francey S, Harrigan S, Patton GC, Jackson HJ: Prediction of psychosis. A step towards indicated prevention of schizophrenia. Br J Psychiatry 1998;172:S14-20.

-41 Behrens TE, Woolrich MW, Jenkinson M, Johansen-Berg H, Nunes RG, Clare S, Matthews PM, Brady JM, Smith SM: Characterization and propagation of uncertainty in diffusion-weighted mr imaging. Magn Reson Med 2003;50:1077-1088.

42 Nichols TE, Holmes AP: Nonparametric permutation tests for functional neuroimaging: A primer with examples. Hum Brain Mapp 2002;15:1-25.

43 Smith SM, Nichols TE: Threshold-free cluster enhancement: Addressing problems of smoothing, threshold dependence and localisation in cluster inference. Neuroimage 2009;44:83-98.

44 Mori S, Wakana S, Nagae-Petscher L, van Zijl P: Mri atlas of human white matter. Elsevier: Amsterdam, 2005; 284 pp.

45 O'Donnell LJ, Pasternak 0: Does diffusion mri tell us anything about the white matter? An overview of methods and pitfalls. Schizophr Res 2015;161:133-141.

-46 Szeszko PR, Robinson DG, Ashtari M, Vogel J, Betensky J, Sevy S, Ardekani BA, Lencz T, Malhotra AK, McCormack J, Miller R, Lim KO, Gunduz-Bruce H, Kane JM, Bilder RM: Clinical and neuropsychological correlates of white matter abnormalities in recent onset schizophrenia. Neuropsychopharmacology 2008;33:976-984.

47 Kanaan R, Barker G, Brammer M, Giampietro V, Shergill S, Woolley J, Picchioni M, Toulopoulou T, McGuire P: White matter microstructure in schizophrenia: Effects of disorder, duration and medication. Br J Psychiatry 2009;194:236-242.

-48 Guo W, Liu F, Liu Z, Gao K, Xiao C, Chen H, Zhao J: Right lateralized white matter abnormalities in firstepisode, drug-naive paranoid schizophrenia. Neurosci Lett 2012;531:5-9.

-49 Alba-Ferrara LM, de Erausquin GA: What does anisotropy measure? Insights from increased and decreased anisotropy in selective fiber tracts in schizophrenia. Front Integr Neurosci 2013;7:9.

50 Bartzokis G, Lu PH, Nuechterlein KH, Gitlin M, Doi C, Edwards N, Lieu C, Altshuler LL, Mintz J: Differential effects of typical and atypical antipsychotics on brain myelination in schizophrenia. Schizophr Res 2007;93:13-22.

51 Fusar-Poli P, Nelson B, Valmaggia L, Yung AR, McGuire PK: Comorbid depressive and anxiety disorders in 509 individuals with an at-risk mental state: Impact on psychopathology and transition to psychosis. Schizophr Bull 2014;40:120-131. 